\title{
NUMERICAL EVALUATION OF CURVATURE EFFECTS ON SHEAR STRESSES ACROSS ARTERIAL STENOSES
}

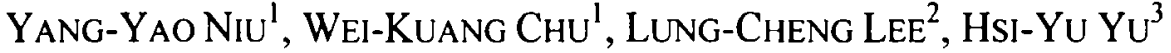 \\ ${ }^{9}$ Institute of Mechanical \& Aerospace Engineering, Chung Hua University, Hsin-Chu \\ ${ }^{2}$ National Center for the High-Performance Computing Center, Hsin-Chu, \\ ${ }^{3}$ National Taiwan University Hospital Taipei, Taiwan
}

\begin{abstract}
In this study. Newtonian flows passing through three-dimensional curved and straight axissymmetrical stenotic tubes are investigated. The geometry effects and Reynolds numbers of 100, 200, 400 , and 600 on the formation of the shear rate over arterial walls are studied. It is noted that geometric effects on flow features such as velocity profiles, pressure and wall shear stress distributions in the post-stenotic region are significant. The location of maximum wall shear stress is found to relate to the geometric effect much than the Reynolds number effect.
\end{abstract}

Biomed Eng Appl Basis Comm. 2002 (August); 14: 164-170.

Keywords: Stenosis, Newtonian fluid curved effects, Shear Stress

\section{INTRODUCTION}

It is well known that aortic dissection, stenosis and aneurysm are the most widely seen among the vascular diseases. Although there have been amazing advances in diagnostic and therapeutic measurements for vascular diseases in the past years, such as in medical imaging technology and interventional measures like balloon angioplasty. However, some fundamental difficulties in the diagnosis and treatment of vascular disorders still remain. For example, the re-stenosis phenomena of arteries after balloon angioplasty are still the unsolved problems. There are considerable evidences that vascular fluid dynamics play an important role in the development and progression of arterial stenosis. It has been noted that blood flow characteristics in arteries are altered significantly by arterial stenosis. The altered hemodynamics may further influ-

Received: May 22, 2002; Accepted: June 25, 2002

Correspondence: Yang-Yao Niu, Professor

Institute of Mechanical and Aerospace Engineering Chung Hua University

No. 30, Tung Shing Road, Hsin Chu, Taiwan

E-mai: yniu@chu.edu.tw ence the development of the disease and arterial deformity, and change the regional blood rheology [1]. Understanding of stenotic flow has been started from theoretical and computational steady-state flow analysis by many researchers [2-8]. For aortic stenosis, most theoretical analysis has regarded the blood as a Newtonian fluid. The assumption of Newtonian behaviors of blood is valid for high shear rate flow in the case of flow through large arteries. It is also found that the flow patterns depend strongly on the geometry of the stenosis and the upstream Reynolds number. For a very severe stenosis with large Reynolds number, It is observed that for flow in tubes with axis-symmetric constrictions of $65 \%$ and $75 \%$ area reductions, flow in the post-stenotic region will become turbulent. Subsequently, pulsatile nature of the blood flow is considered in the further more physiologically realistic models such as [9-19]. It is shown that the post-stenotic flows was became unstable and exhibited trains of counter-rotating vortices which resulted in intense fluctuations in wall shear stress. It is widely accepted that the wall stress distribution of the pulsatile flow plays an important role in the formation of a stenotic artery. However, the previous computation is mostly focused on a straight axis-symmetric tube model. The results presented in these papers show only the change of the separated region, the velocity distribution, the 
resistance and the wall shear stress; however, the influence of curvature effects of stenosis on pressure, the wall shear stress is not studied. Thus, that leaves much room to be investigated.

The main purpose of this study is to improve our understanding of geometry effects at physiologically realistic flow conditions in axis-symmetrical stenosed tubes under including four different Reynold's number $100,200,400$, and 600 . We first study the effect of curved stenoses on wall shear stress distributions, also compared with the results of straight stenotic tubes. In general, the stenotic flow is regarded as a high velocity jet generated from the narrowest section and flow separation away from the stenosis which characterizes flow through constricted tubes. The model is capable of predicting the hemodynamics features most interesting to the physiologists: the detailed pictures of the velocity profile in the separated regions, the pressure drops as well as the wall shear stresses across the stenosis.

\section{NUMERICAL MODEL}

In the mathematical description of arterial stenoses models, the symmetric stress tenser $\sigma_{\imath}, i, j=$ 1, 2, 3 assumed for the Newtonian fluid is expressed by the constitutive equation $\sigma_{i j}=-p \delta_{y j}+2 \mu \mathrm{e}_{1 "}$ with $p$ is the fluid pressure, $\delta$, the Kronecker delta, $\mu$ denotes the apparent fluid viscosity and $\mathrm{e}_{y}, \mathrm{i}, \mathrm{j}=$ $1,2,3$, are the components of the rate-of-strain tensor defined as

$$
\mathbf{e}_{i J}=\frac{1}{2}\left(\frac{\partial u_{i}}{\partial x_{j}}+\frac{\partial u_{j}}{\partial x_{t}}\right) .
$$

Also, the Newtonian flow model applies threedimensional, time-dependent, incompressible NavierStokes equations without body forces in nondimensional form as

$$
\begin{aligned}
& \frac{\partial u_{j}}{\partial x_{1}}=0 \\
& \frac{\partial u_{l}}{\partial t}+u_{,} \frac{\partial u_{l}}{\partial x_{1}}+\frac{\partial p}{\partial x_{i}}=\frac{1}{\operatorname{Re}} \frac{\partial}{\partial x_{1}} \frac{\partial u_{t}}{\partial x_{1}}
\end{aligned}
$$

where $\operatorname{Re}=U_{0} L_{0} / v$ is the Reynolds number by means of the reference length $\mathrm{L}_{0}$, the reference velocity $\mathrm{U}_{0}$ and the kinematic viscosity $v=\mu / \rho$. And $u_{1}, \mathrm{i}=1,2,3$, are the components of the velocity vector and $\rho$ is the constant fluid density.

The 3-D time-dependent Navier-Stokes equations are solved by means of a commercial CFD package CFX4. It adopts the SIMPLE type finite volume method with the third-order accurate QUICK differ- encing scheme for all equations. The flow was assumed to be laminar, incompressible and Newtonian, and the walls were rigid with no slip conditions. The boundary of the flow domain consists of two sections with different types of boundary conditions.At the inlet boundary, a constant uniform velocity profile is used. The pressures at the inlet can be obtained from the solution of the governing equation (3) by assuming the constant velocity inside. At the outflow boundary, the condition describing the surface traction force can be assumed

$$
\left(-p \delta_{\imath j}+\mu\left(\frac{\partial u_{i}}{\partial x_{j}}+\frac{\partial u_{\jmath}}{\partial x_{1}}\right)\right) n_{j}=0
$$

where $n, j=1,2,3$, denote the components of the outward pointing normal unit vector at the outflow boundary. In rigid wall calculations traction-free outflow is assumed.

\section{RESULTS AND DISCUSSION}

CFD simulations are carried out on four different Reynolds numbers of $100,200,400$, and 600 . Velocity and wall shear stress distributions are obtained for the entire flow domain. In this section, flow features such as axial velocity profiles, pressure and wall shear stress distribution around the stenosis section are presented. First of all, Figure 1 demonstrates that the geometry of the straight axis-symmetrical stenotic tube and computational grids for the stenosis models are generated based on a two-block structure with 400,000 volume cells. The non-dimensional geometrical parameters are as follows: inlet diameter 1 ; pre-stenosis length 5 , and stenosis length $1 ; \mathrm{a}$ long post-stenosis domain 31 are chosen in order to minimize the influence of downstream boundary conditions. In order to avoid considering the turbulence effects, a mild stenosis with only $50 \%$ area reduction is considered. Axial velocity profiles of Reynolds number $=100,600$ are selected to be shown in Figure.2. It is shown that the reverse flow distal to the stenosis is not obvious at the case of Reynolds number $=100$. However, the re-circulation region becomes large when the $\mathrm{Re}$ is increased to 600 . It can be noted that the mild stenosis with the small Reynolds number $(\mathrm{Re})$ does not cause significant disturbances distal to the stenotic region, but the mild stenosis model with high-Re flow contains significant flow disturbance phenomena. Next, the pressure distributions along the stenotic region for four different Reynolds number with 100, 200, 400, and 600 are shown in Figure 4 . It is shown that the pressure drop occurs around the neck of the stenotic region no matter what the value of Reynolds number is. A negative pressure difference is increased with the increasing Reynolds number. Furthermore, the estimations of wall shear 

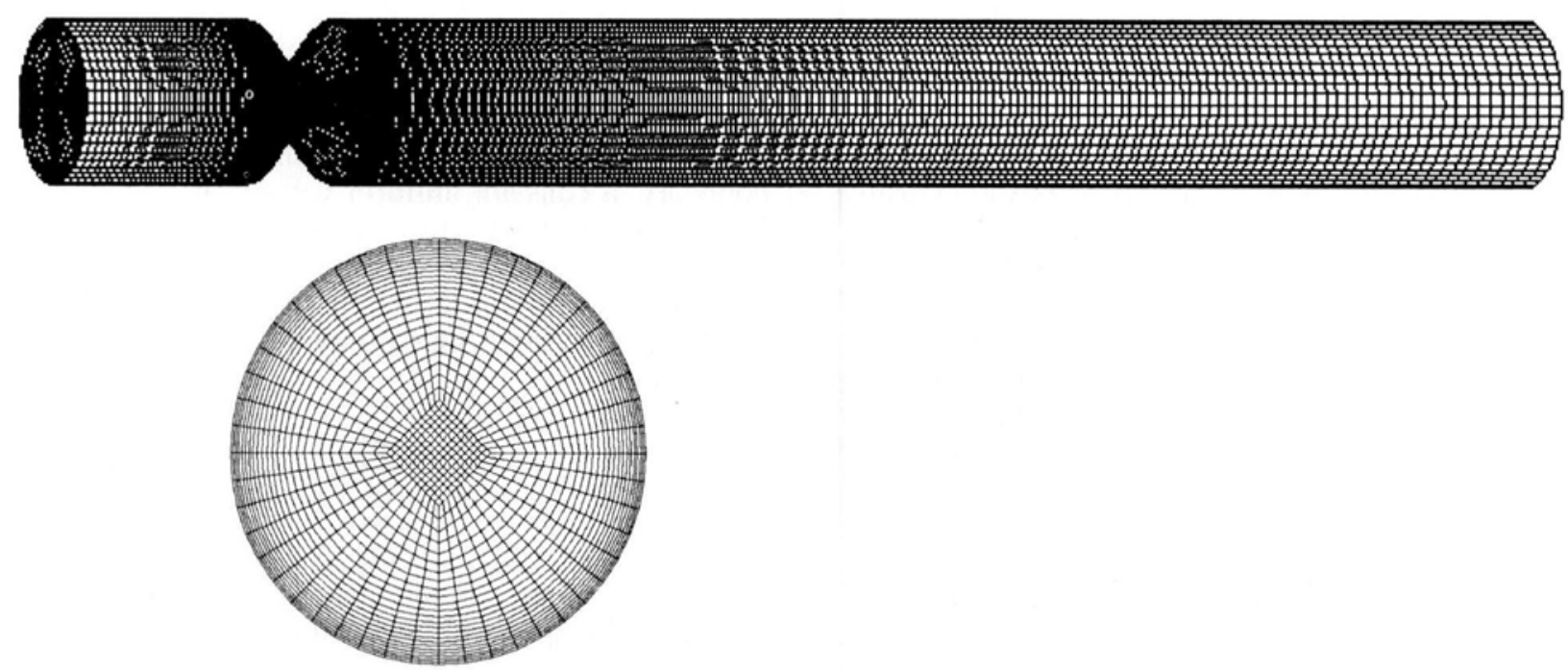

Fig. 1 Geometry \& computational grids for a straight stenotic tube

stress distributions for the whole wall and the stenotic area are depicted in Figure 5 and 6, respectively. It is very encouraging to find that the prediction of the location of maximum wall shear stress is consistent with the analytic studies in [5] which demonstrates the location of maximum wall shear stress is always upstream of the neck of the stenosis and independent of Reynolds number. Excepting that a strong negative shear stress appearing along the throat of the stenosis, no obvious oscillatory shear distributions are found on other downstream wall in the current steady flow model.

Secondly, a two-block grid with the same computational volume cells and the same length and location of the stenosis to the entrance are applied on a curved

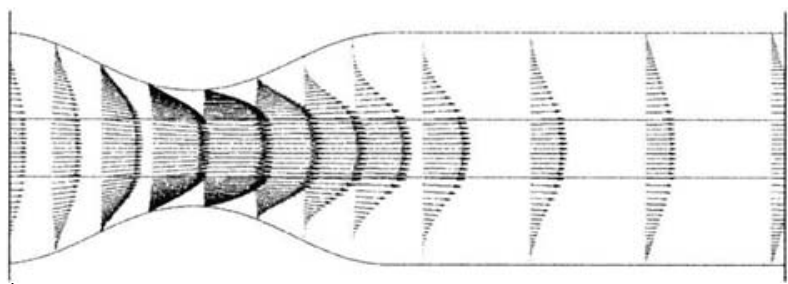

(a) Reynolds No. $=100$

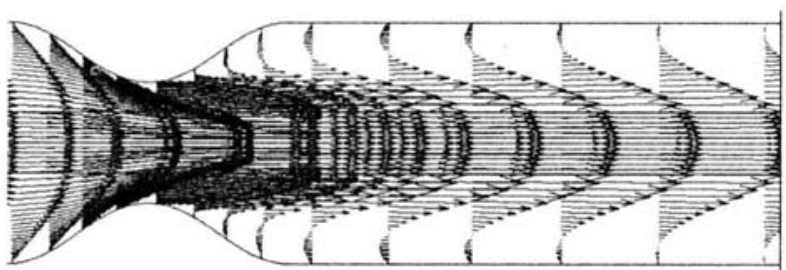

(b) Reynolds No. $=600$

Fig. 2 Vector distributions around the stenosis in a symmetrical stenotic tube axis-symmetrical model shown in Figure 7. Flow features such as axial velocity profiles, pressure and wall shear stress distribution around the stenosis section are also carried out on four different Reynolds numbers of $100,200,400$ and 600 . Figure 7 shows that the axial velocity profiles around the stenosis area by the spiral axis-symmetrical model at the medial plane for Reynolds number $=100,600$. For the case with Reynolds number $=600$, the stenosis has a stronger effect on the post-stenotic flow than the result of Reynolds number $=10$. The pressure and wall stress distributions along the stenotic region for four difference Reynolds's number with $100,200,400$, and 600 are shown

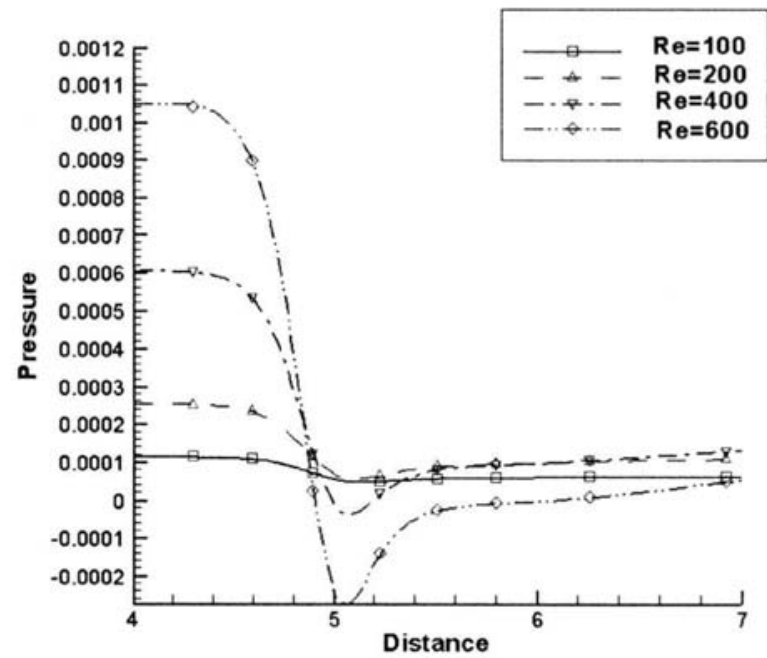

Fig. 3 Variations of pressure at wall for Reynolds numbers $: 100 ; 200 ; 400 ; 600$ : from $X=4$ to $X=7$ 


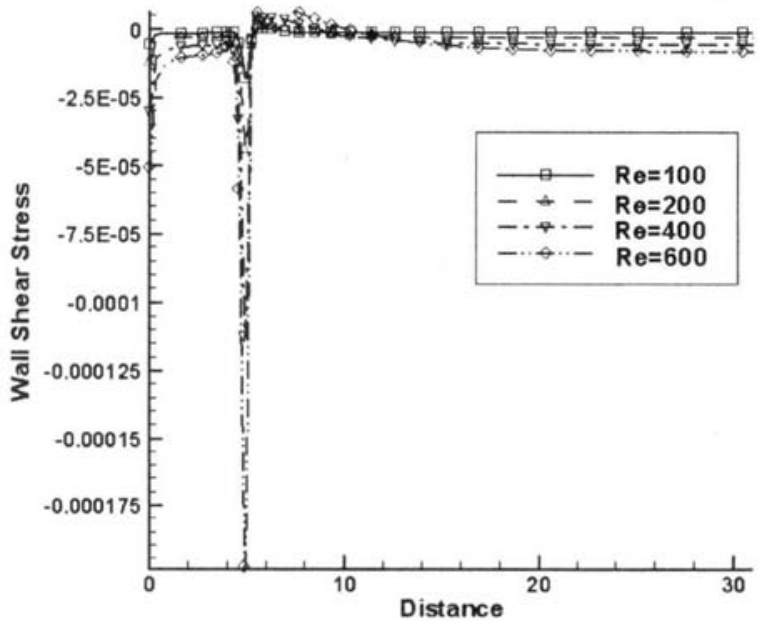

Fig. 4 Variations of wall shear stress at wall for Reynolds numbers $: 100 ; 200 ; 400 ; 600$, From $X=4$ to $X=7$

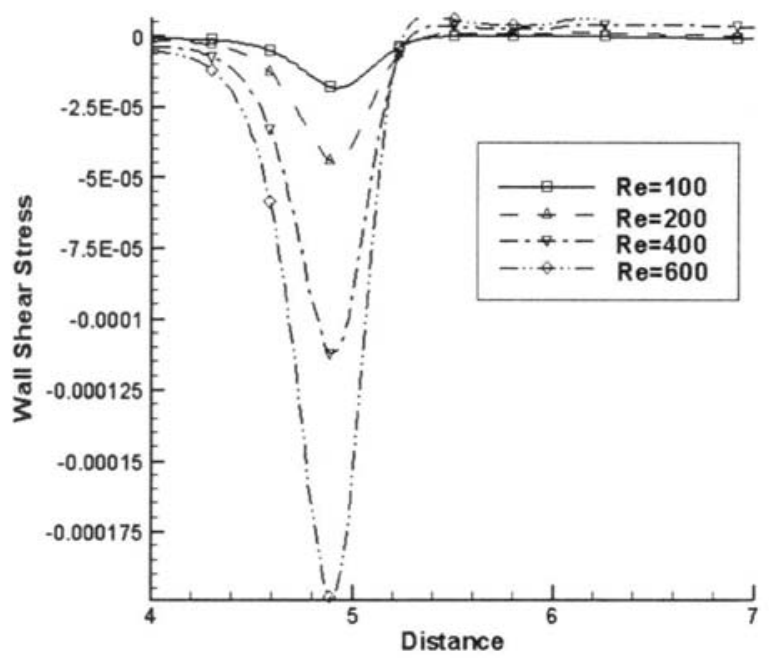

Fig. 5 Variations of wall shear stress at wall for Reynolds numbers $: 100 ; 200 ; 400 ; 600$ From $X=4$ to $X=7$

from Figure 8 to 13. To compare the curved axissymmetrical model with the straight axis-symmetrical model, it is demonstrated that the curved stenotic tube with the pressure drop and the amplitude of the wall stress distribution oscillation in the axial direction is larger than that in the corresponding straight axissymmetrical model. It is noteworthy that the largest positive-negative oscillation occurs closer to the throat of the stenotic region stenosis in all curved stenotic tubes. However, a subsequent strong positive-negative oscillation of wall shear stress behind of the stenotic

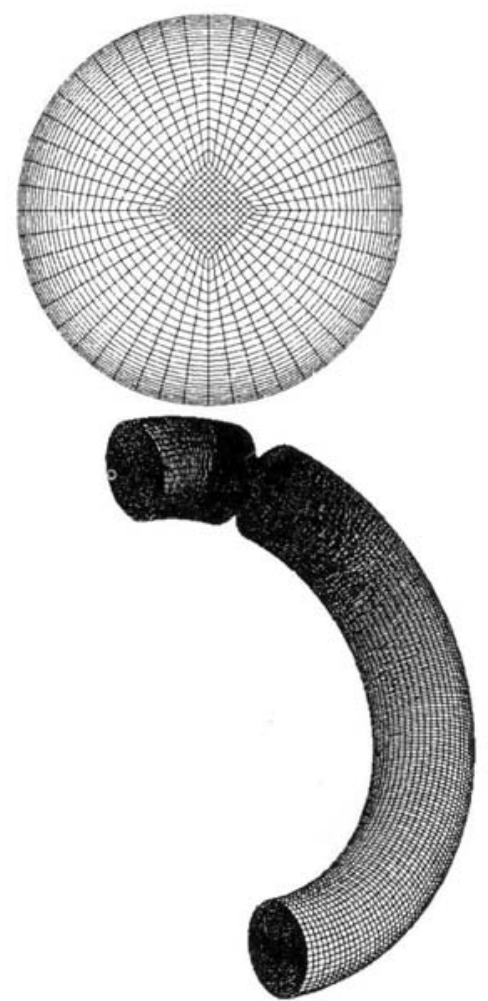

Fig. 6 Geometry \& computational grids for a curved stenotic tube

area are found in the case of $\operatorname{Re}=400$ and 600 on the outer wall. These may correspond to the reattachment locations of the second separation zone behind the stenosis on the outer wall. Furthermore, an earlier pressure drop and negative maximum wall stress are found to occur at the upstream of the stenosis on the inner wall than the locations on the outer wall. It may relate to a weaker adverse pressure gradient existing along the downstream of the inner wall.

\section{CONCLUSION}

In this study, numerical results for velocity profiles and wall shear stress distributions have been obtained for straight and curved stenosis models with mild area reduction. Numerical simulations were performed for idealized cylindrical stenosis geometry with the assumptions including laminar flow, rigid wall, and Newtonian fluid. The results have demonstrated that the formation of shear stress and pressure in the stenotic region by the curved stenosis model are much complex than by straight stenosis model. In the straight stenotic tube, The location of maximum wall shear stress is found to independent of the Reynolds number effect. In the curved stenotic tube, the subsequent strong positive-negative oscillations of wall shear stresses behind the stenotic area are found in the 

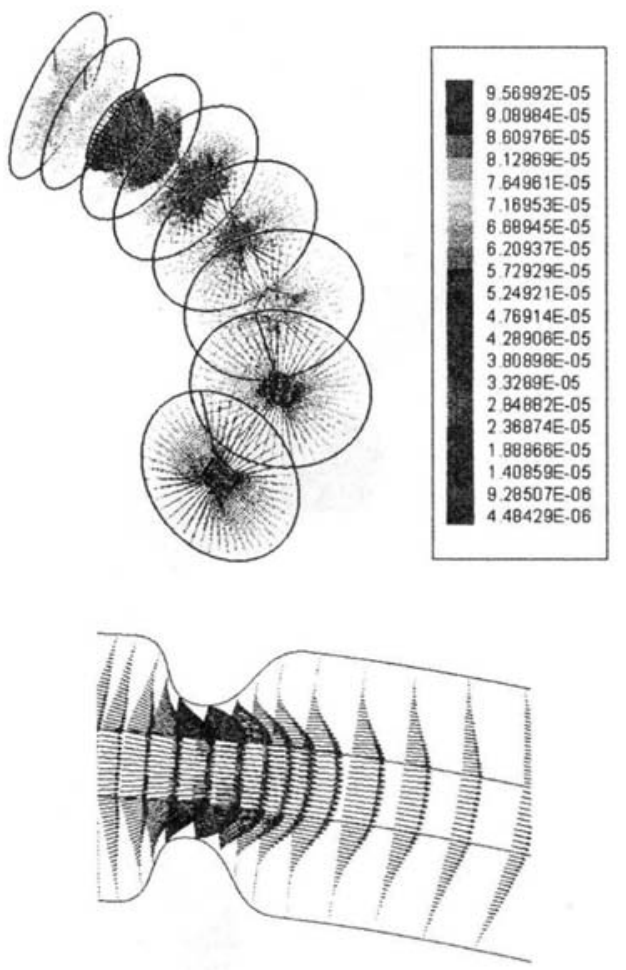

(a) Reynolds No. $=100$
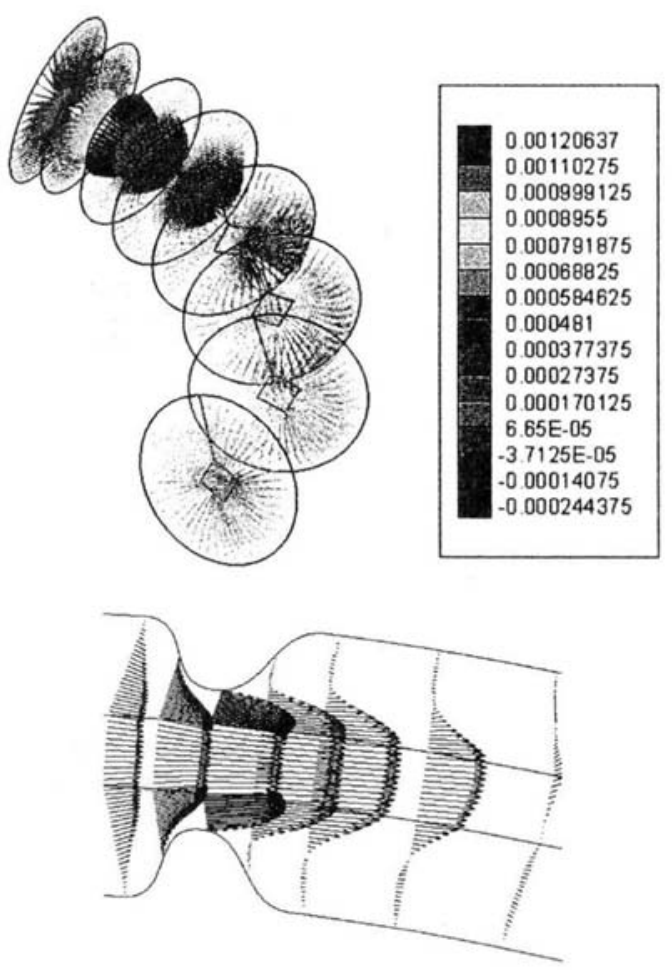

(b) Reynolds No. $=600$

Fig. 7 Velocity vector distributions around the curved stenotic region (the upper one is from the global view, the lower one is from the close view)

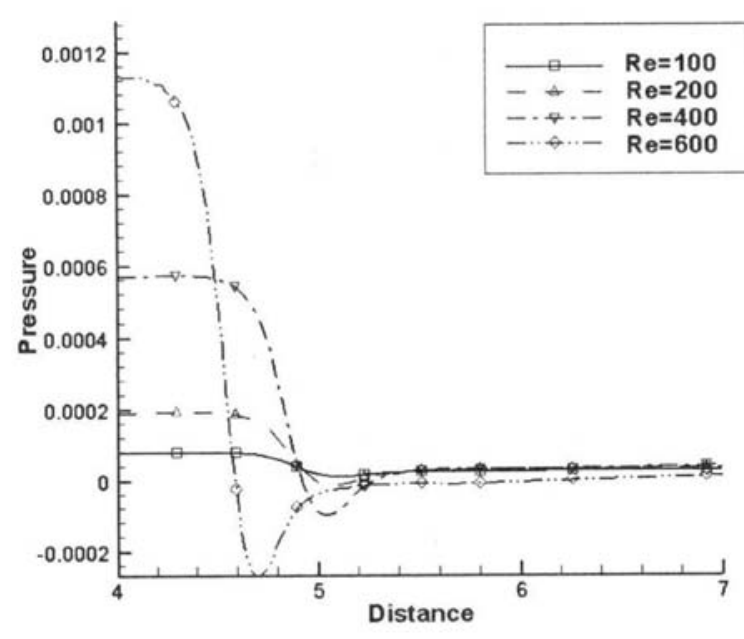

Fig. 8 Variations of Pressure for Reynolds numbers : $100 ; 200 ; 400 ; 600$ From $X=4$ to $X=7$ along the inner wall

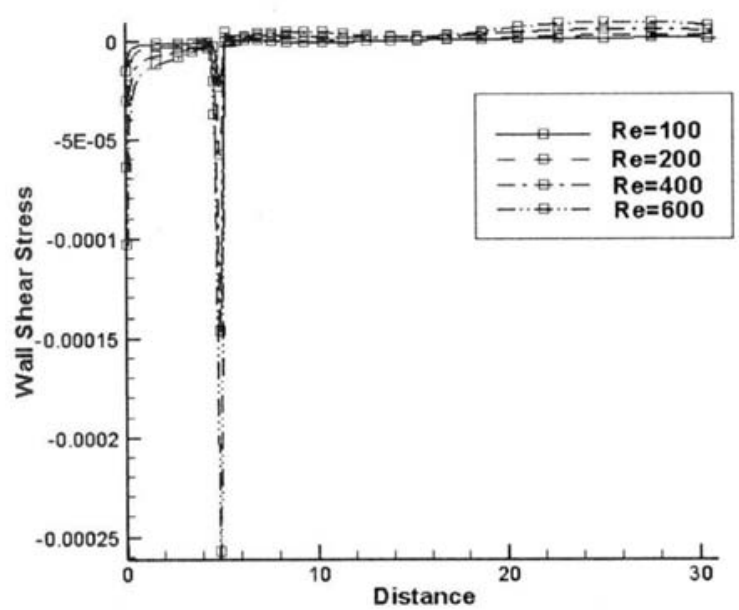

Fig. 9 Variations of wall shear stress for Reynolds numbers : $100 ; 200 ; 400 ; 600$ From $X=0$ to $X=31$ along the inner wall 


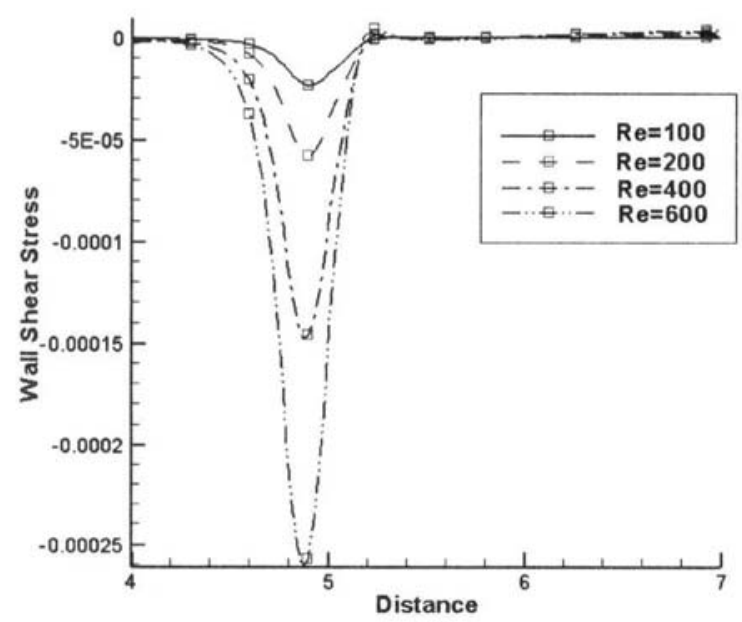

Fig. 10 Variations of wall shear stress for Reynolds numbers $: 100: 200: 400: 600$, From $X=4$ to $X=7$ along the inner wall

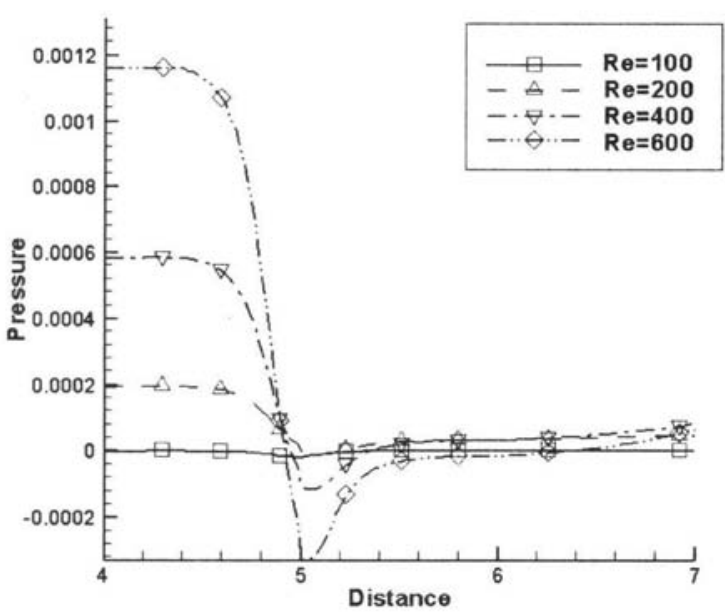

Fig. 11 Variations of pressure for Reynolds numbers : $100: 200 ; 400 ; 600$

From $X=4$ to $X=7$ along the outer wall

case of $\operatorname{Re}=400$ and 600 on their outer wails. Aiso an earlier pressure drop and negative maximum wall stress are found to occur at the upstream of the stenosis on the inner walls. These may correspond to the reattachment locations of the second separation zone behind the stenosis and stronger adverse pressure gradients on the outer wall. The curvature effects on the arterial stenosis by the pulsatile flow model will be considered in future study.

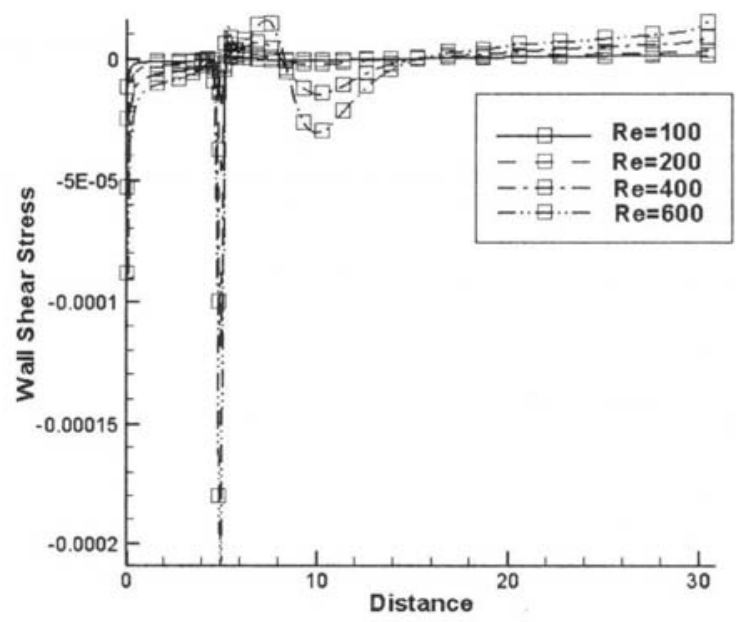

Fig. 12 Variations of wall shear stress for Reynolds numbers : $100: 200: 400: 600$, From $X=0$ to $X=31$ along the outer wall.

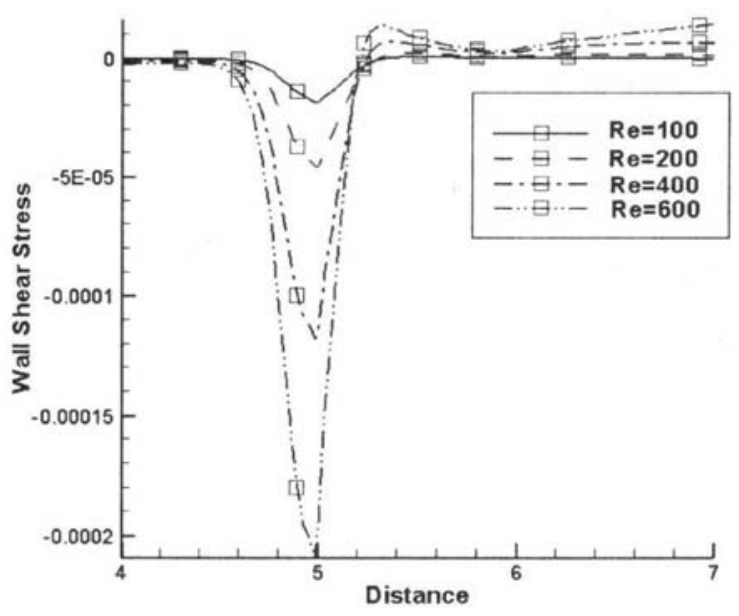

Fig. 13 Variations of wall shear stress for Reynolds numbers : $100: 200: 400: 600$ From $X=4$ to $X=7$ along the outer wall

\section{ACKNOWLEDGEMENT}

The author wishes to acknowledge the computing facility of National Center for the High-Performance Computing, Taiwan. The first author also thanks for the discussions from Dr. Jaw-Lin Wang of Institute of Biomedical Engineering, National Taiwan University on this work. 


\section{REFERENCES}

1. Smith, F.T., "The separation flow through a severely constricted symmetric tube. Journal of Fluid Mechanics" 90, 725-754.,1979

2. Young, D.F., Tsai, F.Y., "Flow characteristics in models of arterial stenosis 1. Steady flow. Journal of Biomechanics "6,395-410., 1973

3. Cheng, L. C, Robertson, J. M. and Dark, M. E. "Numerical calculations of plane oscillatory nonuniform flow II. Parametric study of pressure gradient and frequency with square wall obstacles" J. Biomechanics 6, 521-538, 1973

4. Lee, J. and Fung, Y. "Flow in locally constricted tubes at low Reynolds number". J. Appl, Mech. 37,9-16,1970

5. Morgan B. E. and Young DF., "Am integral method for the analysis of flow in arterial stenosis", Bull. Math. Biol. Vol. 36:39-53, 1974.

6. Deshpande, M.D., Giddens, D.P., Mabon, F.R., "Steady laminar flow through modelled vascular stenoses" Journal of Biomechanics9, 165-174, 1976.

7. Daly, B, J."A numerical study of pulsatile flow through stenosed canine femoral arteries", J. Biomechanics 9, 465-475, 1976

8. McDonal, D. A. "On steady flow through modelled vascular stenoses", J, Biomechanics 12, 13-20,1979

9. Fukushima, T., Azuma, T. and Matsuzawa, T.,'Numerical analysis of blood flow in the vertebral artery" J. Biomech Engng 10, 143-147, 1982

10. Cheng, L. C, Robertson, J. M. and Clark, M. E. "Calculations of plane pulsatile flow past wall obstacles", Computers and Fluids 2,363-380, 1974
11.Lion, R. J., Clark, M. E., Robertson, J. M. and Cheng, L. C. "Three-dimensional simulation of steady flow past a partial stenosis", J. Biomechanics $14,325-337,1981$

12. Kawaguti, M. and Hamano, "Numerical study on post-stenotic dilatation", .Biorheology 20, 507518,1983

13. Tutty, O.R., "Pulsatile flow in a constricted channel", Journal of Biomechanical Engineering Transactions of ASME 114, 50-54,1993

14.Tu, C., Deville, M., Dheur, L., Vanderschuren, L., "Finite element simulation of pulsatile flow through arterial stenosis,",Journal of Biomechanics $25,1141-1152,1993$

15. Cheng Tu and Michel Deville"Pulsatile Flow of Non-Newtonian Fluids through Arterial Stenoses", J. Biomechanics, Vol.29, No.7, pp. 2899-908, 1996

16. Rappitsch, G., Perktold, K., "Pulsatile albumin transport in large arteries: a numerical simulation study," Journal of Biomechanical Engineering Transactions of the ASME 118, 511-519,1993

17. Depiano, E., Siouffi, M., "Experimental and numerical study of pulsatile flows through stenosis: wall shear stress analysis," Journal of Biomechanics 32,1081-1090,1999

18.Long Q., Xu X. Y., Ramnarine, and Hoskins, P. 'Numerical Investigation of Physiologically Realistic Pulsatile Flow through Arterial Stenoses", J. Biomechanics, Vol.34, No.7, pp. 1229-1241, 2001.

19. Tanya Shipkowitz, V.G.J. Rodgers, Lee J. Frazin and K.B. Chandran 'Numerical study on the effect of secondary flow in the human aorta on local shear stresses in abdominal aortic branches", J. Biomechanics, vol.33, pp. 717-728, 2000 